\title{
Optimal Selection of Airport Runway Configurations
}

\author{
Dimitris Bertsimas, Michael Frankovich, Amedeo Odoni \\ Operations Research Center, Massachusetts Institute of Technology, Cambridge, Massachusetts 02139 \\ \{dbertsim@mit.edu,mfrankov@mit.edu, arodoni@mit.edu\}
}

\begin{abstract}
We present a mixed integer programming (MIP) model to solve the problems of (i) selecting an airport's optimal sequence of runway configurations and (ii) determining the optimal balance of arrivals and departures to be served at any moment. These problems, the runway configuration management (RCM) problem and the arrival/departure runway balancing (ADRB) problem, respectively, are of critical importance in minimizing the delay of both in-flight and on-the-ground aircraft along with their associated costs. We show that under mild assumptions on the time required to change between configurations, large realistic problem instances can be solved within several seconds. Furthermore, as assumptions are relaxed, optimal solutions are still found within several minutes. Comparison with a sophisticated baseline heuristic reveals that in many cases the potential reduction in cost from using the method is significant and could be expected to be of the order of at least $10 \%$. Finally, we present an extension of the MIP model to solve these two problems for a group of airports in a metropolitan area such as New York (metroplex), where operations at each airport within the metroplex might have an impact on operations at some of the other airports due to limitations in shared airspace.
\end{abstract}

Subject classifications: transportation: air traffic; programming: integer; applications.

Area of review: Transportation.

History: Received November 2009; revisions received August 2010, January 2011; accepted April 2011. Published online in Articles in Advance November 18, 2011.

\section{Introduction}

The combination of runways that are active at any particular time at an airport is known as the "runway configuration" in which the airport operates at that time. The sequence of configurations selected by controllers greatly influences an airport's capacity to serve demand for arrivals and departures. This paper is concerned with determining a sequence of runway configurations and the assignment of arrivals and departures to the active runways that together minimize the cost incurred at the airport due to delayed aircraft.

Figure 1 shows a schematic representation of Boston's Logan International Airport (BOS) with its six runways. The runway configuration shown in use in the figure consists of three active runways, shown in bold, with the other three being idle. The direction of operations on each is indicated. Two of the runways are used for both arrivals and departures, while the third is used only for departures. For multi-runway airports, the number of possible configurations can be large. For instance, BOS typically employs more than 20 different configurations during a year by making use of its six runways in different ways.

The availability of certain configurations at most major airports might be restricted by the weather conditions prevailing at any particular time. For example, a runway cannot be operated in the presence of strong crosswinds (relative to the orientation of that runway); or a runway might not be adequately instrumented for operations under poor visibility conditions. There are also physical limits to the capacity of each configuration, i.e., to the number of arrivals and departures that can be accommodated in a given length of time while operating in a given configuration. In an operational context, capacity typically is measured as the expected number of movements that can take place in the presence of continuous demand. This is also known as the maximum throughput capacity and is measured as the number of arrivals and departures per unit of time, typically 10,15 , or 60 minutes. In making their decisions concerning the best runway configuration to use at any given time, controllers take into account the capacity of each available configuration, as well as the scheduled demand for arrivals and departures, and the weather forecast, which influences the future availability of the different runway configurations.

In our model, we represent the capacity of different configurations through the runway configuration capacity envelope (RCCE), studied and used in Gilbo (1993, 1997), Hall (1999), and Gilbo and Howard (2000) and discussed in depth in de Neufville and Odoni (2003). An RCCE is a concave piecewise linear function that defines the set of feasible operating points that can be achieved under a given configuration and under given weather conditions. A runway configuration typically will have more than one RCCE, each corresponding to different weather conditions. Figure 2 shows two typical RCCE that could describe the capacity for arrivals and departures of a runway configuration like that shown in Figure 1. Note that the horizontal and vertical axes show, respectively, the number of departures and arrivals that are demanded or performed during the selected unit of time, be that 10,15 , or 60 minutes. The 
Figure 1. A runway configuration used at BOS, with the corresponding active runways shown in bold.

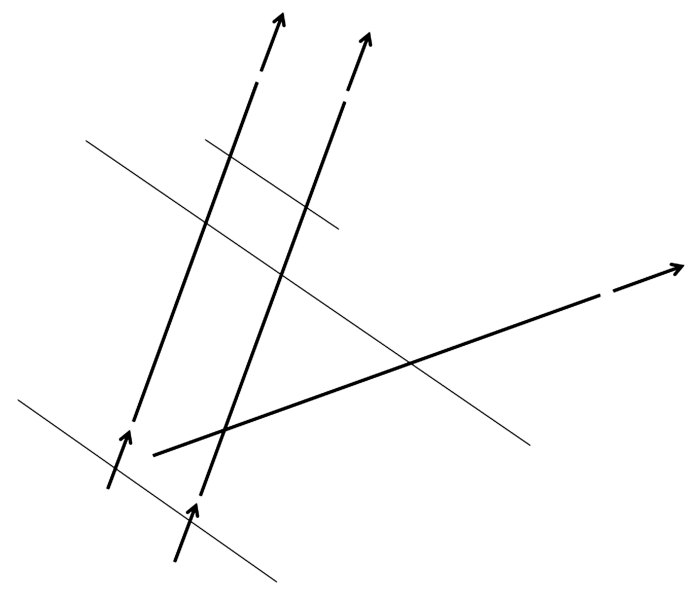

outermost RCCE shows the capacity available under visual meteorological conditions (VMC), while the inner one corresponds to the same configuration under instrument conditions (IMC), when visibility is limited. Any integral point on or within the available RCCE corresponds to a feasible operating point, and any point outside is infeasible. For example, Point 4 is feasible in VMC but infeasible in IMC. In the latter case, the configuration does not have sufficient capacity to accommodate simultaneously the number of arrivals and departures associated with Point 4 during a single unit of time, resulting in the queueing of aircraft. The total number of RCCE that need to be considered, assuming the weather conditions to be known, is equal to the number of configurations, e.g., about 20 in the case of BOS.

In this paper, we present mixed integer programming models (MIP) that solve two problems simultaneously. The first, the runway configuration management (RCM)

Figure 2. Runway configuration capacity envelopes (RCCE) for a single configuration under VMC and IMC.

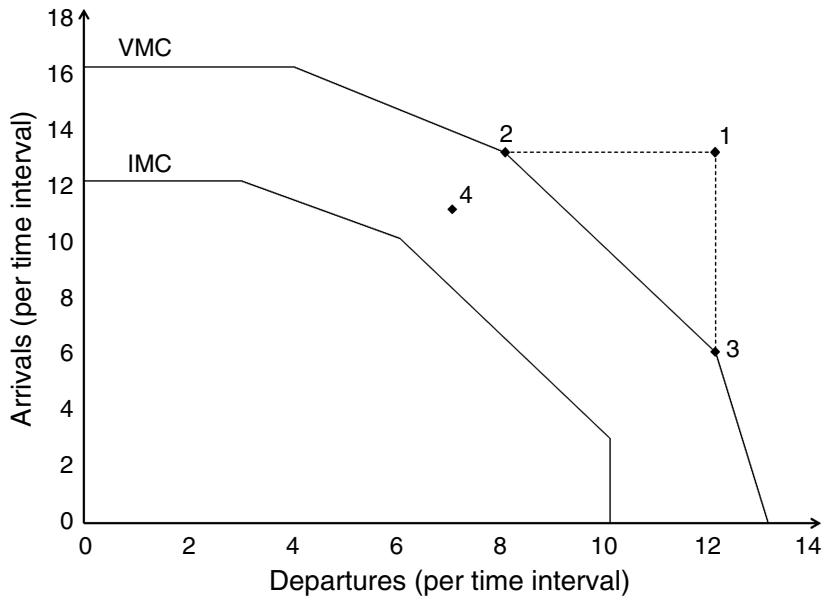

problem, is to find a schedule of runway configurations that will best serve the demand over a specified time horizon. This is the problem we have outlined thus far. Then, given this schedule of configurations, there remains a degree of freedom as to the exact balance of arrivals versus departures to serve during any time interval. Determining the optimal arrival/departure runway balancing (ADRB) is the second problem controllers face. Gilbo (1997) proposed an integer program for the ADRB to minimize queueing by controlling the utilization of near-runway airspace and coordinating operations at arrival and departure fixes subject to a given schedule of configuration use. Furthermore, Gilbo and Howard (2000) proposed an integer program to solve the ADRB problem assuming a constant overall airport RCCE. As far as we are aware, we present here the first optimization approach to the combined RCM and ADRB problems.

In practice, these problems are solved today largely on the basis of experience. Under this essentially "manual" approach, the FAA examines the weather forecast and the expected demand for the next several hours at each major airport and determines the sequence of runway configurations to be used, including the assignment of arrivals and departures to runways for each selected configuration. In doing so, location-specific issues, such as constraints on runway use imposed by noise or other considerations (see §4) are also taken into account. The difficult and most important instances occur when the weather is highly variable or poor (e.g., changing wind directions, occurrence of IMC). In such cases, the combination of changes in runway configurations-with attendant partial or full loss of capacity during changeover times-and of the need to select among "low" RCCE is likely to result in a significant backlog of arrivals and/or departures from one time period to the next. Under such conditions, the situation becomes highly dynamic, and sequences and assignments might be modified frequently in response. The models described here can be most helpful in precisely these cases.

The solution to the RCM and ADRB problems might also impact air traffic flow management (ATFM) actions that the FAA and airlines take in the context of the collaborative decision making (CDM) program. The solution essentially determines the "airport acceptance rate" (AAR) that ATFM uses to specify the number of arrivals that can be scheduled at each airport in each time period, i.e., the number of arrival "slots" that will be allocated under CDM among the airlines using the airport. If the AAR at an airport is low compared to the forecast demand for arrivals there, a ground delay program (GDP) can be initiated by ATFM for that airport, thus delaying before take-off (at their originating airport) flights headed to that airport. In this sense, the RCM and ADRB problems have implications not only at the local level but also at a national one.

In solving these problems, our objective is to minimize the total weighted cost of delays to queued aircraft, where the weights might take into account the cost of fuel burn on the airport's surface (for departures) and in the 
near-airport airspace (for arrivals), as well as other considerations (e.g., controller workload) that might result in arrival delays being assigned a higher weight than delays to departures. Note that (i) the changeover times needed to transition between successive runway configurations and (ii) the restricted availability of certain configurations at certain times due to weather conditions are both critical aspects of the optimization to be performed. The former precludes the optimality of a greedy policy that simply operates in the best configuration for the demand in the current time interval (without taking into account the future sequence of configurations), while the latter requires that we consider the "lifespan" of each candidate configuration.

In our models, selecting a configuration is equivalent to selecting the outermost available RCCE corresponding to that configuration, this RCCE being determined by current weather conditions. (We assume that, for a given configuration, the IMC RCCE is always fully contained within the one for VMC.) Selecting the arrival/departure balance level is then equivalent to selecting an integral point on or within this RCCE. This selection also affects how delay, if any, is allocated between arrivals and departures. For instance, in Figure 2, let Point 1 correspond to the demand $\left(d_{1}, a_{1}\right)$ during hour 1 and assume that the airport operates in VMC. Because Point 1 lies outside the available RCCE, some of the demand for the hour in question cannot be accommodated. In this particular instance, an optimal choice of operating point, considering only this hour, clearly lies somewhere on the line connecting Points 2 and 3.

We assume that the break points of each RCCE are integral so that, along with nonnegativity constraints, the RCCE defines the convex hull of its feasible operating points in the two-dimensional departures-arrivals operating space. These RCCE can be obtained by observing past operating points and fitting minimal concave piecewise linear functions which bound these points (see Gilbo 1993 and Kellner and Kösters 2008). Alternatively, computational models of airport operations can be used to generate them, as discussed in Swedish (1981), de Neufville and Odoni (2003), and Stamatopoulos et al. (2004).

\section{Contributions and Outline of Paper}

The most important contribution of this paper is the development of mixed integer programming models to solve the RCM and ADRB problems efficiently for a single airport, as well as the proposed extension of the models to the metroplex case. In particular:

- In $\S 1$, we present a MIP for the single airport problem, assuming that the changeover time between configurations is a constant, $C$, for all pairs of configurations. While this MIP does not define the convex hull of feasible solutions, several computational experiments on large realistic data sets in $\S 1.3$ indicate that the formulation is a strong one. Furthermore, the results of these experiments indicate that the potential benefit of using this approach is significant and that improvements of the order of at least $10 \%$ could be expected relative to a sophisticated optimization-based heuristic.

- In $\S 2$, we relax the above assumption to allow the changeover time between any two configurations to belong to a set of the form $\{i C: i=1, \ldots, \nu\}$, where $C$ is a constant. Computational experiments show that for $\nu \in\{2,3\}$, optimal solutions are still obtained within several minutes.

- In $\S 3$, we present an extension of the model to optimize over a metroplex of airports.

- In $\S 4$, we show that important environmental constraints can be easily incorporated into the model.

\section{A Mixed Integer Programming Model for a Single Airport with Constant Changeover Times}

Here we seek to solve the RCM and ADRB problems, outlined in the introduction, for a single airport. We assume that the time required to change between configurations is a constant, $C$, and that the relevant data, as listed in the definitions below, are available and known with certainty. The latter assumption is reasonable given that we consider in our model a short time horizon of a few hours. Furthermore, we envisage the method being implemented using a rolling horizon approach, re-solving every 30 minutes or so to account for any divergence of observed from expected data.

$\mathscr{T}=$ the set of time intervals, $\{1,2, \ldots, T\}$;

$\mathscr{K}_{t}=$ the set of configurations available during time interval $t$;

$\mathscr{F}_{k}^{t}=$ the set of linear pieces of the outermost RCCE available for configuration $k$ at time $t$;

$a_{t}=$ the number of arrivals scheduled for time interval $t$;

$d_{t}=$ the number of departures scheduled for time interval $t$;

$c_{t}=$ the cost of delaying a single arrival for one time interval at time interval $t$;

$q_{t}=$ the cost of delaying a single departure for one time interval at time interval $t$.

In addition, we let the $j$ th piece of the outermost RCCE available for configuration $k$ at time $t$, an affine function, be defined by the parameters $\alpha_{j k}>0, \beta_{j k} \leqslant 0$, and $\gamma_{j k} \geqslant 0$. While these parameters depend on $t$, because $j$ belongs to a set that depends on $t$, we omit this from our notation.

Note that the time horizon is discretized such that the length of one time interval is equal to the changeover time $C$, which is typically of the order of 10 minutes. In this way, we can model a changeover by allowing no arrivals or departures to be served during the time interval at which the changeover occurs. Moreover, the set of time intervals, $\mathscr{T}$, includes a number of time intervals at the end of the original time horizon, with no scheduled arrivals or departures. During these added time intervals, any queues that have built up can be cleared. We assume that we are 
able to clear all queues without having to cancel or re-route any flights.

Our decision variables are the following and are defined for every $t$ only for those configurations $k$ that are available:

$z_{k t}= \begin{cases}1, & \text { if we operate in configuration } k \text { at time } t, \\ 0, & \text { otherwise; }\end{cases}$

$y_{k t}=\left\{\begin{array}{l}\text { the number of arrivals served at time } t, \\ \quad \text { if we operate in configuration } k \text { at time } t, \\ 0, \quad \text { otherwise; }\end{array}\right.$

$x_{k t}=\left\{\begin{array}{l}\text { the number of departures served at time } t, \\ \quad \text { if we operate in configuration } k \text { at time } t, \\ 0, \quad \text { otherwise; }\end{array}\right.$

$u_{t}=$ the number of arrivals which go unserved at time $t$;

$v_{t}=$ the number of departures which go unserved at time $t$.

The mixed integer optimization problem is as follows:

$$
\begin{array}{ll}
\text { P1: } \min & \sum_{t \in \mathscr{T}}\left(c_{t} u_{t}+q_{t} v_{t}\right) \\
\text { s/t. } & u_{t}-u_{t-1}+\sum_{k \in \mathscr{K}_{t}} y_{k t}=a_{t}, \quad \forall t \in \mathscr{T}, \\
& v_{t}-v_{t-1}+\sum_{k \in \mathscr{K}_{t}} x_{k t}=d_{t}, \quad \forall t \in \mathscr{T}, \\
& \gamma_{j k} y_{k t}-\beta_{j k} x_{k t}-\alpha_{j k} z_{k t} \leqslant 0, \\
& \quad \forall j \in \mathscr{F}_{k}^{t}, \forall k \in \mathscr{K}_{t}, \forall t \in \mathscr{T}, \\
& \sum_{k \in \mathscr{K}_{t}} z_{k t} \leqslant 1, \quad \forall t \in \mathscr{T}, \\
& \sum_{k^{\prime} \in \mathscr{K}_{t-1} \backslash\{k\}} z_{k^{\prime}, t-1}+z_{k t} \leqslant 1, \\
& \\
& z_{k t} \in\{0,1\}, \quad \forall k \in \mathscr{K}_{t}, \forall t \in \mathscr{T}, \\
& u_{t}, v_{t} \in \mathbb{Z}_{+}, \quad \forall t \in \mathscr{T}, \\
& y_{k t} \geqslant 0, \quad \forall k \in \mathscr{K}_{t}, \forall t \in \mathscr{T}, \\
& x_{k t} \geqslant 0, \quad \forall k \in \mathscr{K}_{t}, \forall t \in \mathscr{T}, \\
& u_{0}, v_{0}=0 . \quad
\end{array}
$$

Our objective is to minimize the total cost of delays incurred. Constraints (1), (2), (7), and (10) define the variables $u_{t}$ and $v_{t}$, the number of arrivals and departures, respectively, that go unserved at time $t$. Constraints (3) force the operating point, given that we operate in the $k$ th configuration at time $t$, to lie within its RCCE, while forcing it to zero if $z_{k t}=0$. Constraints (4) state that at any time $t$, we may operate in at most one configuration.

Constraints (5) invoke our fundamental assumption that the changeover time is equal to the length of one time interval. In this way, we model the cost of changing from one configuration to another by enforcing a delay of one time interval. In other words, it is not possible to operate in configuration $k$ at time $t$ and also in configuration $k^{\prime} \neq k$ at time $t-1$.

One could also modify Constraints (5) to consider only a subset of all pairs of configurations $\left\{k, k^{\prime}\right\}$, thus modeling the changeovers between the excluded pairs as being instantaneous. This would be suitable if the corresponding changeover times were very small compared to other changeovers.

Note that we have defined the variables $u_{t}$ and $v_{t}$ to be integral in Constraints (7). Given our assumptions on the nature of the RCCE and the integrality of the arrival and demand data, as well as Constraints (6) on $z_{k t}$, it follows that in an optimal solution to the resulting MIP, $y_{k t}$ and $x_{k t}$ are integral. We therefore relax the integrality constraints for these two sets of variables and greatly reduce the number of integer variables in the model.

We can then add two classes of valid inequalities to strengthen the formulation. First, we add Inequalities (11) below, which are closely related to Constraints (5). Observe that in Inequalities (11), we require for all $k$ that we cannot both operate in configuration $k$ at time $t-1$ and also in some configuration $k^{\prime} \neq k$ at time $t$, while in Constraints (5), we require for all $k$ that we cannot both operate some configuration $k^{\prime} \neq k$ at time $t-1$ and in configuration $k$ at time $t$.

$z_{k, t-1}+\sum_{k^{\prime} \in \mathscr{K}_{t} \backslash\{k\}} z_{k^{\prime} t} \leqslant 1, \quad \forall k \in \mathscr{K}_{t-1}, \forall t \in \mathscr{T} \backslash\{1\}$.

It is clear that while the set of feasible integer solutions remains unchanged under this addition, we remove some nonintegral solutions from the solution set of the LP relaxation of P1.

Now we generate for each time interval $t$ a single RCCE that defines the convex hull of the set $\left\{(x, y) \in \mathbb{Z}_{+}^{2}\right.$ : $\exists k \in \mathscr{K}_{t}$ s.t. $\left.\gamma_{j k} y-\beta_{j k} x \leqslant \alpha_{j k}, \forall j \in \mathscr{F}_{k}^{t}\right\}$, i.e., the minimal piecewise linear concave envelope that majorizes all RCCE at time $t$. We let this RCCE be defined by the parameters $\alpha_{j t}^{\prime}, \beta_{j t}^{\prime}$, and $\gamma_{j t}^{\prime}, \forall j \in \mathscr{J}_{t}^{\prime}$. Then, observing that $y_{t} \triangleq \sum_{k \in \mathscr{K}_{t}} y_{k t}$ and $x_{t} \triangleq \sum_{k \in \mathscr{K}_{t}} x_{k t}$ represent the number of arrivals and departures served at time $t$, respectively, it is clear that $\left(x_{t}, y_{t}\right)$ must lie within this RCCE, and hence Inequalities (12) below are valid.

$\gamma_{j t}^{\prime} \sum_{k \in \mathscr{K}_{t}} y_{k t}-\beta_{j t}^{\prime} \sum_{k^{\prime} \in \mathscr{K}_{t}} x_{k^{\prime} t} \leqslant \alpha_{j t}^{\prime}, \quad \forall j \in \mathscr{J}_{t}^{\prime}, \forall t \in \mathscr{T}$.

Furthermore, these inequalities give the tightest possible bound on the relation between feasible $y_{t}$ and $x_{t}$, because the convex hull of this (in general, nonconvex) set has been defined.

\subsection{Example Problem}

We now present a simple example to test the model and gain insight into its solution. We consider a time horizon 
Figure 3. Test Problem 1 data, with three configurations and 15 time intervals.

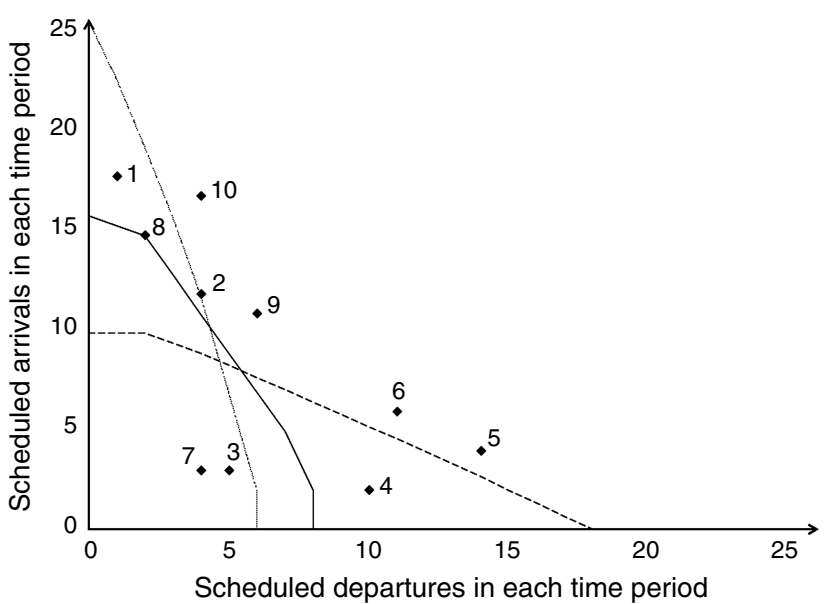

Notes. RCCE 1 has a solid line, RCCE 2 a dotted line, and RCCE 3 a dashed line. The scheduled demand is indicated for intervals 1 to 10 and is zero for intervals 11 to 15 .

of 10 periods of demand, set $T=15$ to allow time to serve all demand, and $c_{t}=12, q_{t}=10, \forall t \in \mathcal{T}$ to capture the typically greater cost of delaying arrivals compared to departures. In all examples that follow, we shall use these cost coefficients. The scheduled demand for arrivals and departures is displayed in Figure 3 along with the RCCE corresponding to three configurations, which are available throughout the entire time horizon.

This problem, along with all others that follow in this paper, was solved using AMPL CPLEX 11.2.10, using a single thread, on a computer with an Intel(R) Core(TM) 2 Duo E7400 Processor $(2.80 \mathrm{GHz}, 3 \mathrm{MB}$ Cache, $1,066 \mathrm{MHz}$ FSB) and 2 GB of RAM, running Ubuntu Linux. The optimal solution, which is displayed in more detail in Figures 4,5 , and 6 , was found in 0.04 seconds and consists of operating in configuration 2 for intervals 1 and 2, configuration 3 for intervals 4 to 6 , and then in configuration 2 again from interval 8 onward.

Note that the "actual demand" in the system at time $t$, given the operating policy in the preceding time intervals, consists of $u_{t-1}+a_{t}$ arrivals and $v_{t-1}+d_{t}$ departures, i.e., any arrivals (departures) left in queue at the end of time interval $t-1$ plus the scheduled arrivals (departures) in time interval $t$. We can then observe the following:

1. In Figure 4, the actual demand and scheduled demand are identical because the first demand point lies within RCCE 2, with which we operate, and hence all demand is served and there is no backlog added to the second time interval.

2. In Figure 5, because we are changing from configuration 2 to configuration 3 , we have $x_{3}=y_{3}=0$. In this way, the third actual demand point is added to the fourth scheduled demand point to create the fourth actual demand point. Similar behavior is also seen with time intervals 7 and 8 in Figure 6.
Figure 4. Test Problem 1 solution, intervals 1 and 2.

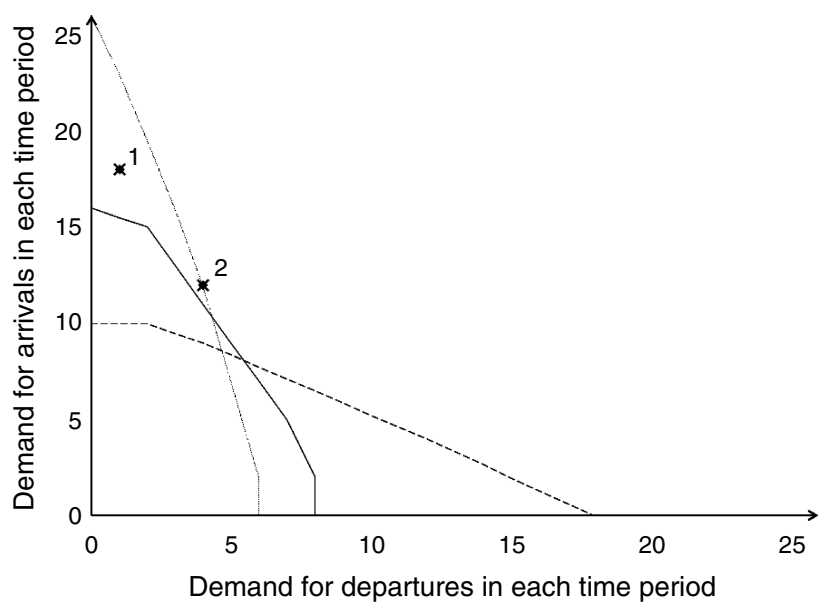

Notes. RCCE 1 has a solid line, RCCE 2 a dotted line, and RCCE 3 a dashed line. Scheduled demand is indicated by a diamond, actual demand by a cross, although these are identical here.

3. When a change is made, it is made during an interval of relatively low actual demand and before a sequence that is favored by the new configuration.

4. As time passes, the scheduled and actual demand could diverge significantly.

In summary, we have learned that even for a very small problem, it is essential to account for the cumulative nature of demand and its dependence on our decisions; our early decisions might have a long-lasting impact on the overall problem. These interactions might be very complex, and hence good decision-making will in general be difficult without the use of sophisticated tools.

\subsection{A Baseline Policy}

Before performing computational experiments on problems of realistic size, we now devote our attention to presenting

Figure 5. Test Problem 1 solution, intervals 3 to 6 .

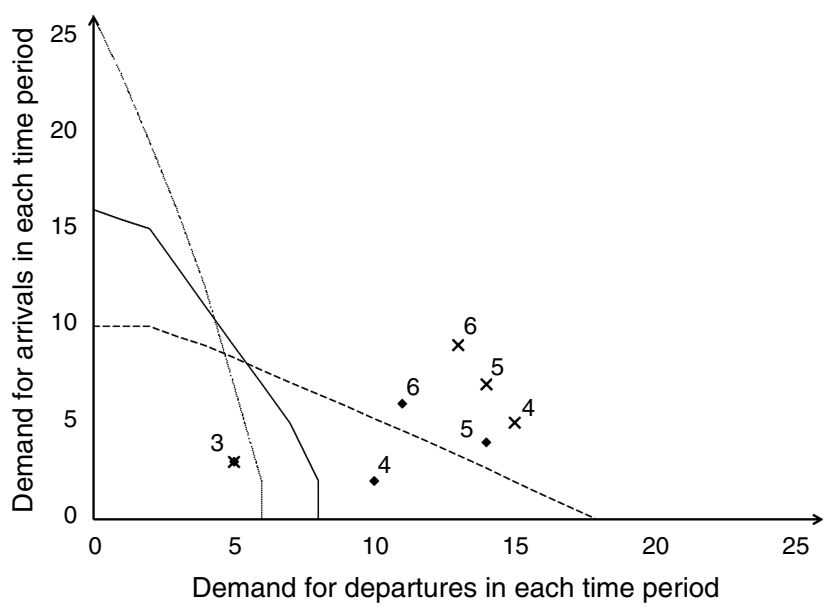

Notes. RCCE 1 has a solid line, RCCE 2 a dotted line, and RCCE 3 a dashed line. Scheduled demand is indicated by a diamond, actual demand by a cross. 
Figure 6. Test Problem 1 solution, intervals 7 to 12 .

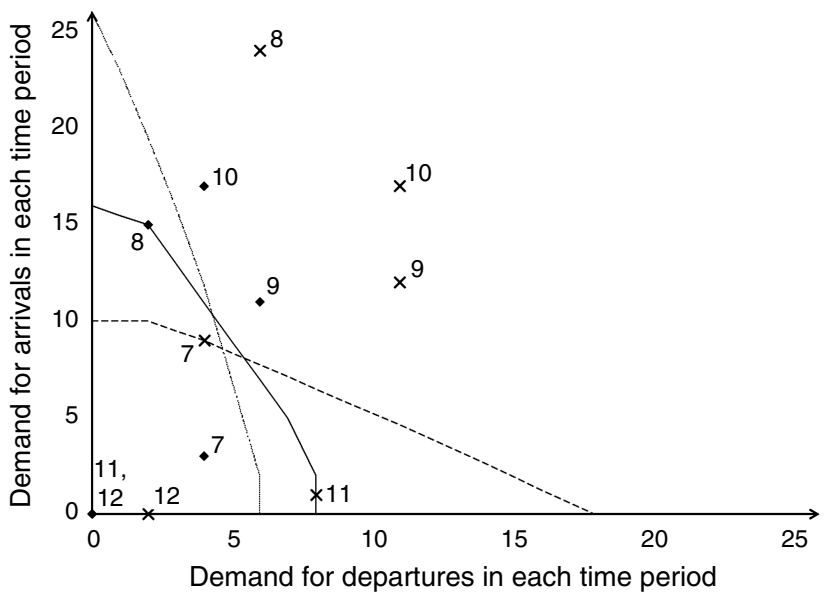

Notes. RCCE 1 has a solid line, RCCE 2 a dotted line, and RCCE 3 a dashed line. Scheduled demand is indicated by a diamond, actual demand by a cross.

a baseline policy in order to obtain a reasonable indication of the relative quality of the solutions obtained from P1 compared with, say, current practice. The baseline policy was designed (i) to obtain an estimate of a lower bound on the improvement that could be observed in practice by implementing the policy obtained from solving P1 and (ii) to mimic the approach that might be taken by a highly skilled controller. The method is developed through a "smart" heuristic, summarized below first at a high level and then in detail.

1. Among all configurations that are available for a significant period of time starting now, choose the one that is best (per the criteria described below) to operate in.

2. Operate in this configuration until the first time period at which it is no longer available for use.

3 . When that happens, observe the new state of the system: if there is no demand left, then stop; otherwise, return to Step 1.

The algorithm is essentially a greedy algorithm, modified to avoid a large number of costly changeovers. We believe this to be a good approximation of controllers' actions: given the difficulty of foreseeing the effect of one's decisions on future demand, one cannot plan "manually" a good configuration sequence too far into the future.

To choose which configuration is selected as "best," we first restrict our list of eligible configurations to those that are available for a reasonably long period of time, preventing too many forced changeovers. We then solve a relevant optimization problem, $\mathrm{P} 2(t)$, shown below, over these configurations. $\mathrm{P} 2(t)$ is an advanced optimization problem and, as a result, the RCM and ADRB policies that are developed by the baseline heuristic are sophisticated. We are confident that, on average, any improvement in objective obtained through P1 over a policy resulting from the baseline heuristic will represent a lower bound on the improvement that would be observed in practice by implementing a policy obtained from P1.
In making such a claim, one cannot ignore the stochastic environment in which this problem is solved. Recall, however, that we consider a short time horizon of a few hours, limiting the uncertainty associated with the data. Moreover, implementation of our methodology should use a rolling horizon approach, re-solving the MIP every 15 minutes or so, to take into account changing conditions while always looking far enough ahead into the future to avoid simply relying on a greedy policy.

The following algorithm computes and simulates the baseline policy.

\section{Algorithm 1}

1. Set $t:=1$, and let $f:=0$ be the simulated cost, $\bar{a}_{\tau}:=a_{\tau}$ the actual demand for arrivals and $\bar{d}_{\tau}:=d_{\tau}$ the actual demand for departures, $\forall \tau \in \mathscr{T}$. Also let $\eta$ be the default number of consecutive time periods for which a configuration must be available to be considered for selection. In this paper, we let $\eta=6$, corresponding to one hour.

2. If $\mathscr{K}_{t} \neq \varnothing$, then go to Step 3; else, no demand is served at this time period, so update the simulated cost and actual demand by setting $f:=f+c_{t} \bar{a}_{t}+q_{t} \bar{d}_{t}, \bar{a}_{t+1}:=$ $\bar{a}_{t+1}+\bar{a}_{t}, \bar{d}_{t+1}:=\bar{d}_{t+1}+\bar{d}_{t}$, and $t:=t+1$. If $t \leqslant T$, then go to Step 2; else, Stop.

3. Let $\overline{\mathscr{K}}_{t}=\bigcap_{h=0}^{\eta-1} \mathscr{K}_{t+h}$ be the set of configurations eligible for selection. If $\mathscr{K}_{t}=\varnothing$, then set $\eta:=\eta-1$ and go to Step 3; else, set $\eta:=6$ and go to Step 4.

4. Given the state of the system, choose the configuration to operate in by solving the MIP $\mathrm{P} 2(t)$ if $t=1$, or $\mathrm{P} 2(t+1)$ otherwise:

$$
\begin{array}{ll}
\mathbf{P 2}(\mathbf{t}): \quad \min & \sum_{\tau=t}^{T}\left(c_{\tau} u_{\tau}+q_{\tau} v_{\tau}\right) \\
\mathrm{s} / \mathrm{t} . \quad & u_{\tau}+\sum_{k \in \overline{\mathscr{H}}_{t}} y_{k \tau}=a_{\tau}, \quad \forall \tau \in\{t, \ldots, T\}, \\
& v_{\tau}+\sum_{k \in \overline{\mathscr{H}}_{t}} x_{k \tau}=d_{\tau}, \quad \forall \tau \in\{t, \ldots, T\}, \\
& \gamma_{j k} y_{k \tau}-\beta_{j k} x_{k \tau}-\alpha_{j k} z_{k \tau} \leqslant 0, \\
& \forall j \in \mathscr{F}_{k}^{t}, \forall k \in \overline{\mathscr{H}}_{t}, \quad \forall \tau \in\{t, \ldots, T\}, \\
& z_{k \tau}-\theta_{k} \leqslant 0, \quad \forall k \in \overline{\mathscr{H}}_{t}, \\
& \quad \forall \tau \in\{t, \ldots, T\}, \\
& \sum_{k \in \overline{\mathscr{H}}_{t}} \theta_{k} \leqslant 1, \quad \forall k \in \overline{\mathscr{H}}_{t}, \\
& z_{k \tau} \in\{0,1\}, \quad \forall \tau \in\{t, \ldots, T\}, \\
& u_{\tau}, v_{\tau} \in \mathbb{Z}_{+}, \quad \forall \tau \in\{t, \ldots, T\}, \\
& y_{k \tau} \geqslant 0, \quad \forall k \in \overline{\mathscr{H}}_{t}, \forall \tau \in\{t, \ldots, T\}, \\
& x_{k \tau} \geqslant 0, \quad \forall k \in \overline{\mathscr{K}}_{t}, \forall \tau \in\{t, \ldots, T\} .
\end{array}
$$

Note that Constraints (16) and (17) mean that only one configuration can be chosen for the entire time horizon $t, \ldots, T$. Furthermore, only scheduled demand is 
considered for any given time period, i.e., the backlogging of demand is not taken into account in Constraints (13) and (14), unlike in the related Constraints (1) and (2) of P1. The underlying rationale is that working with scheduled demand, rather than going through the difficult process of calculating actual demand, more closely resembles the realworld decision-making approach of controllers (as shown in the example problem of $\S 1.1$, the effect of controllers' decisions on future demand might be very hard to predict, hence this is a good way to model their decision process).

Hence $\mathrm{P} 2(t)$ chooses the single best configuration according to scheduled demand over the remaining time horizon. We let $k^{*}$ be this configuration, i.e., such that $\theta_{k^{*}}^{*}=1$, where $\boldsymbol{\theta}^{*}$ is the optimal vector $\theta$ of $\mathrm{P} 2(t)$, and let $t^{\prime}=\min \left\{\tau: \tau>t, k^{*} \notin \mathscr{K}_{\tau}\right\}$ be the first time at which configuration $k^{*}$ becomes unavailable (note that this will be $T+1$ if $k^{*}$ is available for the rest of the time horizon). We will operate in configuration $k^{*}$ until time $t^{\prime}-1$.

5. If $t \neq 1$, then let $\delta:=0$, preventing demand from being served at time $t$ through Inequalities (24) in $\mathrm{P} 3\left(k^{*}, \delta, t, t^{\prime}\right)$ below; else, let $\delta:=1$.

6. Now simulate the effect of this choice of $k^{*}$ for the interval $\left[t, t^{\prime}-1\right]$. Solve the following MIP, $\mathrm{P} 3\left(k^{*}, \delta, t, t^{\prime}\right)$ :

$$
\mathbf{P 3}\left(\mathbf{k}^{*}, \boldsymbol{\delta}, \mathbf{t}, \mathbf{t}^{\prime}\right) \text { : }
$$

$$
\begin{array}{ll}
\min & \sum_{\tau=t}^{t^{\prime}-1}\left(c_{\tau} u_{\tau}+q_{\tau} v_{\tau}\right) \\
\mathrm{s} / \mathrm{t} . & u_{\tau}-u_{\tau-1}+y_{\tau}=\bar{a}_{\tau}, \quad \forall \tau \in\left\{t, \ldots, t^{\prime}-1\right\}, \\
& v_{\tau}-v_{\tau-1}+x_{\tau}=\bar{d}_{\tau}, \quad \forall \tau \in\left\{t, \ldots, t^{\prime}-1\right\}, \\
& \gamma_{j k^{*}} y_{t}-\beta_{j k^{*}} x_{t}-\delta . \alpha_{j k^{*}} z_{t} \leqslant 0, \quad \forall j \in \mathscr{f}_{k^{*}}^{t},
\end{array}
$$

$$
\begin{aligned}
& \gamma_{j k^{*}} y_{k^{*} \tau}- \beta_{j k^{*}} x_{\tau}-\alpha_{j k^{*}} z_{\tau} \leqslant 0, \\
& \forall j \in \mathscr{F}_{k^{*}}^{\tau}, \forall \tau \in\left\{t+1, \ldots, t^{\prime}-1\right\}, \\
& z_{\tau} \in\{0,1\}, \quad \forall \tau \in\left\{t, \ldots, t^{\prime}-1\right\}, \\
& u_{\tau}, v_{\tau} \in \mathbb{Z}_{+}, \quad \forall \tau \in\left\{t, \ldots, t^{\prime}-1\right\}, \\
& y_{\tau} \geqslant 0, \quad \forall \tau \in\left\{t, \ldots, t^{\prime}-1\right\}, \\
& x_{\tau} \geqslant 0, \quad \forall \tau \in\left\{t, \ldots, t^{\prime}-1\right\}, \\
& u_{t-1}, v_{t-1}=0 .
\end{aligned}
$$

The above MIP simply fixes the configuration that is operated over the time interval $\left[t, t^{\prime}-1\right]$ to be $k^{*}$, and simulates the effect of this decision, forcing zero demand to be served at time $t$ if $\delta=0$.

7. Update the simulated cost and actual demand by setting $f:=f+\sum_{\tau=t}^{t^{\prime}-1}\left(c_{\tau} u_{\tau}^{*}+q_{\tau} v_{\tau}^{*}\right), \bar{a}_{t^{\prime}}:=\bar{a}_{t^{\prime}}+u_{t^{\prime}-1}^{*}$, and $\bar{d}_{t^{\prime}}:=\bar{d}_{t^{\prime}}+v_{t^{\prime}-1}^{*}$.

8. Set $t:=t^{\prime}$. If $t \leqslant T$, then go to Step 2 .

9. Stop. The baseline value is $f$.

\subsection{Computational Results for Larger Problems}

Now we introduce several problems of realistic size, shown in Figures 7, 8, and 9. Note that the set of RCCE in Problem 4 is a superset of that in Problem 3, which is itself a superset of that in Problem 2. Each problem has 30 time intervals, representing 24 10-minute intervals, and 6 intervals at the end with zero demand. Table 1 outlines the availability of configurations. In addition, when a configuration is available, some of its RCCE might be unavailable, but these details are omitted for brevity.

Figure 7. Test Problem 2, with four configurations, 11 RCCE, and 30 time intervals.

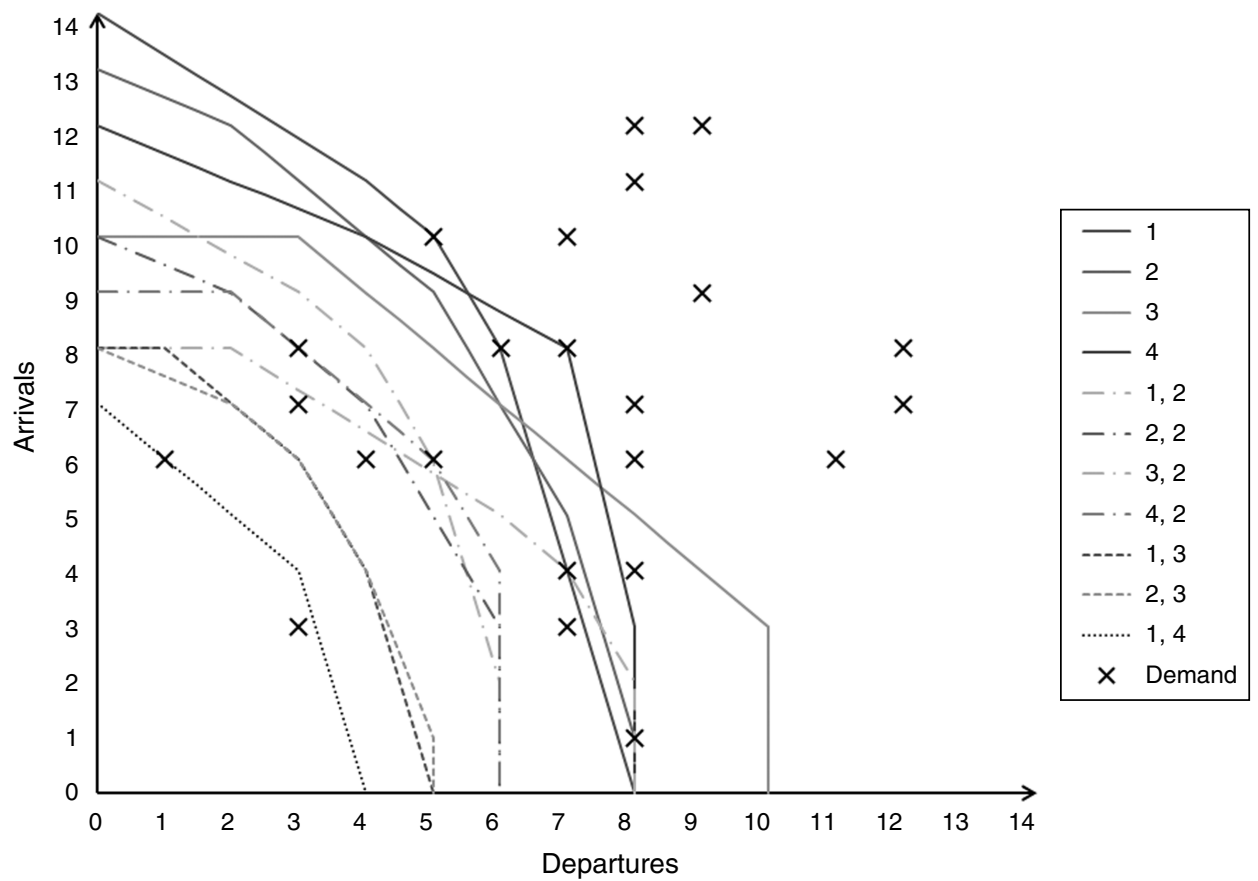


Figure 8. Test Problem 3, with eight configurations, $22 \mathrm{RCCE}$, and 30 time intervals.
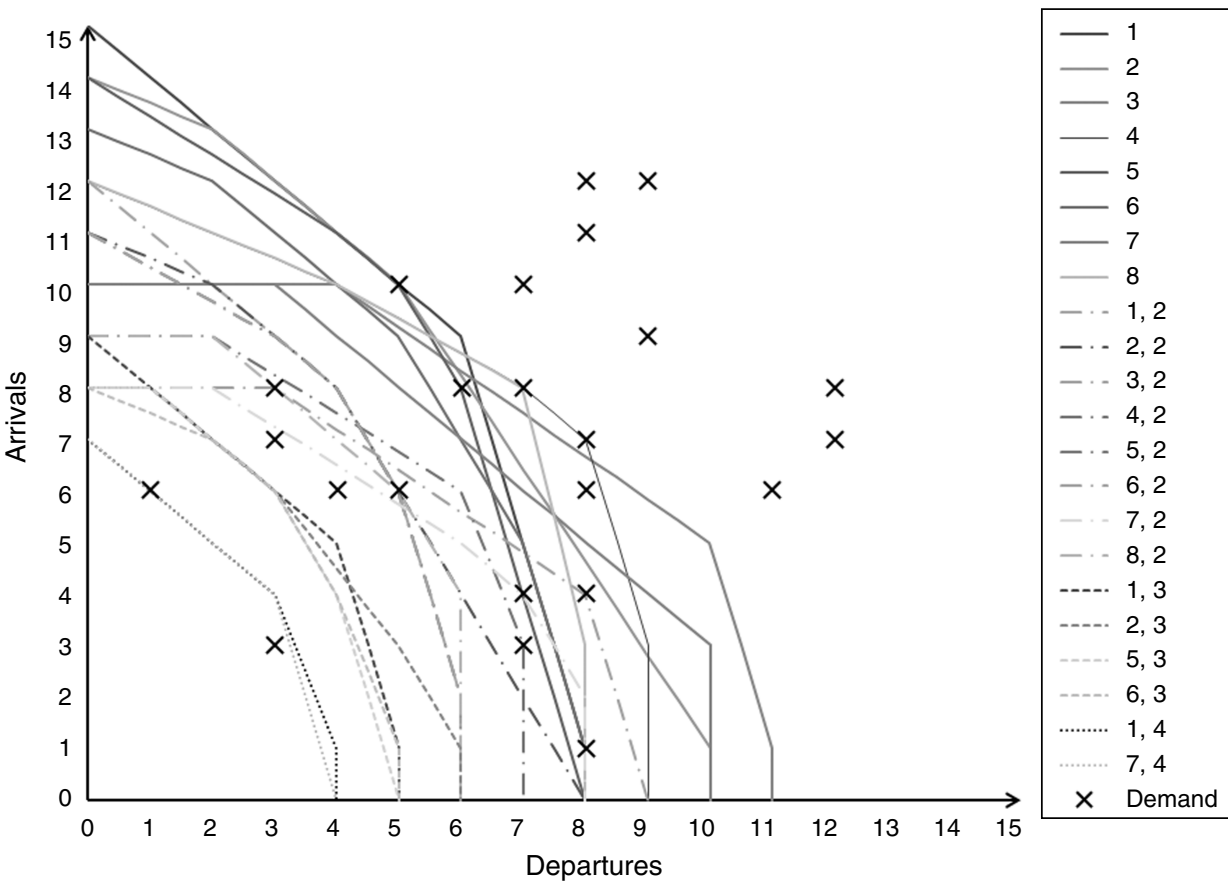

Table 2 shows that computation times were in all cases less than three seconds, even for the largest of problems. Indeed, another indication of the strength of the model, also shown in the table, is the small difference between the optimal objective value of $\mathrm{P} 1, Z_{I P}^{*}$ and the optimal value of the $L P$ relaxation of $\mathrm{P} 1, Z_{L P}^{*}$.

We now compare the policies and objective values obtained from P1 to those of the baseline heuristic. The results are presented in Tables 4 and 5. Note that we have introduced further examples 5-7, with 9,8 , and 9 configurations, respectively, whose detailed description we omit for brevity. We do, however, describe the availability of these RCCE in Table 3.

It can be observed that in many cases our model far outperforms the baseline approach, by margins of $10 \%-50 \%$. This can be explained by the cumulative nature of the demand in the system, which amplifies the negative effect of any suboptimal decisions made early in the time horizon.

Figure 9. Test Problem 4, with 12 configurations, 33 RCCE, and 30 time intervals.

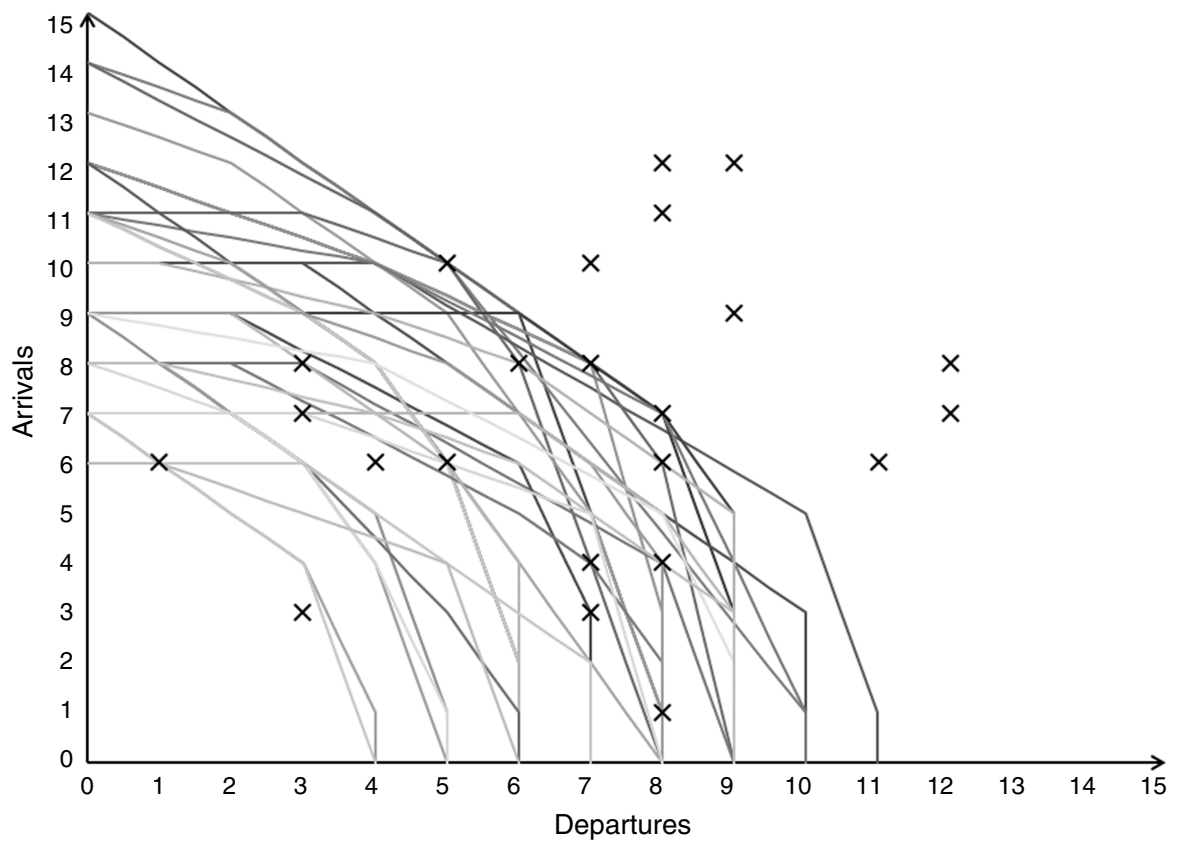


Table 1. Availability of configurations for Problems $2-4$.

\begin{tabular}{lc}
\hline Problem & Configurations that are unavailable \\
\hline 2 & 1 at $t=20 ; 2$ on $[19,21] ; 3$ on $\{6,12,25\} ;$ \\
3 & 4 on $\{5,6,12,13,25\}$ \\
& 1 and 5 on $[16,17] ; 2$ and 6 on $[15,17] ;$ \\
3 and 7 on $\{4,27\} ; 4$ and 8 on $\{4,5,27\}$ \\
4 & $1,5,9$, and 11 on $[16,17] ; 2$ and 6 on $[15,17] ;$ \\
& 3 and 7 on $\{4,27\} ; 4,8$, and 12 on $\{4,5,27\} ;$ \\
& 10 on $\{4,15,27\}$ \\
\hline
\end{tabular}

However, we also note that in one instance where the policies are similar the improvement is only $2 \%$.

A small difference could also be expected when all configurations are available at all time periods, because in this case the baseline heuristic would produce a policy that uses a single configuration, and P1 would also be likely to do the same despite its slight difference from P2(1). However, in this case the heuristic almost becomes a complete optimization. Furthermore, this case is an uninteresting one because in practice many configurations often cannot be operated for prolonged periods of time. This problem becomes most interesting when some configurations become unavailable and we must determine whether to choose the one that is best now, even though it will become unavailable at a later point, or to choose some other configuration that will remain available for a longer period of time. In any case, it is clear that the potential benefit from the implementation of this method could be significant. Overall, we expect to see an average improvement of the order of at least $10 \%$ in practice, given the results presented here, and the advanced nature of our baseline heuristic, which involves solving a relevant optimization problem.

\section{Multiple Changeover Times}

We now extend the model to allow different changeover times belonging to a finite set of the form $\{i C: i=$ $1, \ldots, \nu\}$. We must first discretize the time horizon further, such that the length of one time interval is equal to the minimum changeover time $C$. For a given problem solved by $\mathrm{P} 1$, this will increase the number of time intervals in the time horizon and scale down the RCCE, both by a factor of $\nu$. Note that the new RCCE will then not necessarily define the convex hull of the feasible operating points, hence all such RCCE must be rounded so that they do

Table 2. Computation times for $\mathrm{P} 1$ and a measure of the strength of the formulation.

\begin{tabular}{lcccc}
\hline Problem & $\begin{array}{c}\text { Computation } \\
\text { time (s) }\end{array}$ & $Z_{I P}^{*}$ & $Z_{L P}^{*}$ & $\begin{array}{c}\% \text { difference between } \\
Z_{I P}^{*} \text { and } Z_{L P}^{*}\end{array}$ \\
\hline 2 & 0.1 & 6,148 & $5,681.4$ & 7.6 \\
3 & 1.1 & 4,752 & $3,376.4$ & 28.9 \\
4 & 2.6 & 3,330 & $2,788.7$ & 16.2 \\
\hline
\end{tabular}

Table 3. Availability of configurations for problems 5-7.

\begin{tabular}{|c|c|}
\hline Problem & Configurations that are unavailable \\
\hline 5 & $\begin{array}{l}1 \text { at } 10,17, \text { and } 18 ; 2 \text { at } 21 ; 5 \text { at } 11,20 \text {, and } 21 ; \\
\quad 6 \text { on }[10,12] \text {, at } 20 \text { and } 21 ; 9 \text { at } 21\end{array}$ \\
\hline 6 & $\begin{array}{l}3,4,5,6,7 \text {, and } 8 \text { on }[10,11] ; 1,2,3,4,5 \text {, and } 6 \\
\text { on }[20,21]\end{array}$ \\
\hline 7 & 2,3, and 4 on $[13,14] ; 6,7$, and 8 at 25 \\
\hline
\end{tabular}

indeed satisfy this requirement. However, we cannot make the discretization too fine because the rounding error of these RCCE would then become significant.

To model changeover times for cases in which one time interval now becomes two, we let $\mathscr{K}^{\prime}=\left\{\left(k_{1}, k_{2}\right),\left(k_{3}, k_{4}\right)\right.$, $\left.\ldots,\left(k_{m-1}, k_{m}\right)\right\}$ be the set of ordered pairs representing changeovers that take two time intervals, where $k_{i} \in$ $\bigcup_{t \in \mathscr{T}} \mathscr{R}_{t}, \forall i \in\{1, \ldots, m\}$. We then add the following constraints, of which there are at most $m(T-2) / 2$, to the model

$$
\begin{aligned}
z_{k, t-2}+\sum_{k^{\prime}:\left(k, k^{\prime}\right) \in \mathscr{K}^{\prime}} z_{k^{\prime} t} \leqslant 1, & \\
& \forall k \text { s.t. } \exists\left(k, k^{\prime}\right) \in \mathscr{K}^{\prime}, \forall t \in \mathscr{T} \backslash\{1,2\} .
\end{aligned}
$$

For three changeover times, which for all practical purposes is the most that would be necessary, we first triple the number of time intervals in the time horizon and then add the constraints for those changeovers that take two time intervals, exactly as in (31) above. In addition, we let $\mathscr{K}^{\prime \prime}=$ $\left\{\left(k_{1}, k_{2}\right),\left(k_{3}, k_{4}\right), \ldots,\left(k_{n-1}, k_{n}\right)\right\}$ be the set of ordered pairs representing changeovers that take three time intervals. We then add the following constraints to the model:

$$
\begin{aligned}
z_{k, t-3}+\sum_{k^{\prime}:\left(k, k^{\prime}\right) \in \mathscr{K}^{\prime \prime}} z_{k^{\prime} t} \leqslant 1, & \\
& \forall k \text { s.t. } \exists\left(k, k^{\prime}\right) \in \mathscr{K}^{\prime \prime}, \forall t \in \mathscr{T} \backslash\{1,2,3\} .
\end{aligned}
$$

Finally, we can again add valid inequalities in the spirit of Inequalities (11), corresponding to both Constraints (31) and (32).

\subsection{Size of the Model}

We now present in Table 6 the effect of allowing multiple changeover times on the size of the model. To simplify,

Table 4. Comparison of solutions from the model P1 with baseline solutions.

\begin{tabular}{lccc}
\hline Problem & $\begin{array}{c}\text { P1 solution } \\
\text { value }\end{array}$ & $\begin{array}{c}\text { Baseline } \\
\text { solution value }\end{array}$ & \% difference \\
\hline 2 & 6,148 & 6,964 & 13.3 \\
3 & 4,752 & 7,180 & 51.1 \\
4 & 3,330 & 3,396 & 2.0 \\
5 & 8,462 & 12,510 & 47.8 \\
6 & 8,826 & 10,504 & 19.0 \\
7 & 10,078 & 11,638 & 15.5 \\
\hline
\end{tabular}


Table 5. Comparison of the optimal policies and the baseline policies.

\begin{tabular}{lll}
\hline Problem & \multicolumn{1}{c}{ P1 policy } & \multicolumn{1}{c}{ Baseline policy } \\
\hline 2 & 1 on $[1,17] ; 4$ on $[19,24] ; 3$ on $[26,30]$ & 1 on $[1,19] ; 1$ on $[21,30]$ \\
3 & 1 on $[1,5] ; 4$ on $[7,26]$ & 6 on $[1,14] ; 4$ on $[16,26] ; 4$ on $[28,30]$ \\
4 & 11 on $[1,15] ; 10$ on $[17,26]$ & 11 on $[1,15] ; 4$ on $[17,26]$ \\
5 & 9 on $[1,19] ; 1$ on $[21,30]$ & 1 on $[1,9] ; 1$ on $[11,16] ; 4$ on $[18,30]$ \\
6 & 2 on $[1,19] ; 8$ on $[21,30]$ & 8 on $[1,9] ; 2$ on $[11,19] ; 7$ on $[21,30]$ \\
7 & 2 on $[1,12] ; 6$ on $[14,23] ; 5$ on $[25,30]$ & 3 on $[1,12] ; 8$ on $[14,24] ; 8$ on $[26,30]$ \\
\hline
\end{tabular}

Table 6. Effect of the number of changeover times on the size of the model.

\begin{tabular}{|c|c|c|c|}
\hline Changeover times & $\begin{array}{l}\text { Integer } \\
\text { variables }\end{array}$ & $\begin{array}{l}\text { Continuous } \\
\text { variables }\end{array}$ & $\begin{array}{l}\text { Upper bound on } \\
\text { number of constraints }\end{array}$ \\
\hline 1 & $T(2+K)(420)$ & $2 K T(720)$ & $T(3+J+J K+4 K)(2,700)$ \\
\hline 2 & $2 T(2+K)(840)$ & $4 K T(1,440)$ & $2 T\left(3+J+J K+4 K+2 K^{2}\right)(22,700)$ \\
\hline 3 & $3 T(2+K)(1,260)$ & $6 K T(2,160)$ & $3 T\left(3+J+J K+4 K+4 K^{2}\right)(60,000)$ \\
\hline
\end{tabular}

we assume that $\forall k \in \mathscr{K}_{t}, \forall t \in \mathscr{T}$, we have $\left|\mathscr{K}_{t}\right|=K$ and $\left|\mathscr{F}_{k}^{t}\right|=\left|\mathscr{g}_{t}^{\prime}\right|=J$.

Note that in the worst case, allowing for three changeover times increases the number of constraints by a factor of 20, but the model is still not excessively large. This case occurs when there is a single changeover that is about a third of the length of all others.

\subsection{Computational Results}

In the interest of estimating computation times for the case of multiple changeover times, as well as gaining some insight into the effect of this modeling adjustment on optimal solutions, we next modify the three problems from $\S 1.3$. We first consider the case of two changeover times, allowing the changeovers between two configurations to take half the time of all other changeovers. These shorter changeovers are indicated in Table 7, along with the corresponding optimal policies and computation times.

From Table 7, it can be seen that computation times increased by an order of magnitude over those of Table 2 in $\S 1.3$ but were still very short. Furthermore, different optimal policies are obtained that favor the shorter changeovers, compared to those of Table 5 in $\S 1.3$. In particular, the optimal configuration sequence for Problem 2 changes from $(1,4,3)$ to $(1,3,1)$, while that for Problem 3 changes from $(1,4)$ to $(2,3)$, and that for Problem 4 from $(11,10)$

Table 7. Policies and computation times for two changeover times.

\begin{tabular}{|c|c|c|c|}
\hline Problem & $\begin{array}{c}\text { Short } \\
\text { changeovers }\end{array}$ & Optimal policy & Time $(\mathrm{s})$ \\
\hline $2^{\prime}$ & $\{1,3\}$ & $\begin{array}{l}1 \text { on }[1,35], 3 \text { on }[37,48] \text {, } \\
1 \text { on }[50,56]\end{array}$ & 0.5 \\
\hline $3^{\prime}$ & $\{2,3\}$ & 2 on $[1,7], 3$ on $[9,51]$ & 24.2 \\
\hline $4^{\prime}$ & $\{4,11\}$ & 11 on $[1,18], 4$ on $[20,50]$ & 4.9 \\
\hline
\end{tabular}

to $(11,4)$, each clearly taking advantage of the shorter changeover times, as might be expected.

Next we modify the problems from $\S 1.3$ in a similar way to allow for three different changeover times. The modifications and results are shown in Table 8. Again, the optimal policies change due to the different changeover times between certain configurations. For example, comparing with Table 7, the optimal configuration sequence for Problem 2 changes from $(1,3,1)$ to $(1,3,2)$, given the short changeover between configurations 2 and 3 . Furthermore, the long changeover between configurations 1 and 4 rules out the optimal solution $(1,4,3)$ to the original problem, shown in Table 5, §1.3.

While it is clear that computation times are significantly longer than in the case of one or two changeover times, these results indicate that optimal solutions are obtained quickly enough to warrant successful implementation in practice, even for the largest problems.

\section{Optimizing Over a Metroplex of Airports}

We now extend the problem to consider multiple airports operating within close proximity of one another. This is

Table 8. Policies and computation times for three different changeover times.

\begin{tabular}{lcccr}
\hline Problem & Short & Long & Optimal policy & Time (s) \\
\hline $2^{\prime \prime}$ & $\{2,3\}$ & $\{1,4\}$ & 1 on $[1,52], 3$ on $[55,72]$, & 2.4 \\
& & & 2 on $[74,84]$ \\
$3^{\prime \prime}$ & $\{3,4\}$ & $\{1,4\}$ & 2 on $[1,16], 4$ on $[19,77]$ & 122.1 \\
$4^{\prime \prime}$ & $\{4,10\}$ & $\{10,11\}$ & 11 on $[1,27], 4$ on $[30,76]$ & 44.0 \\
\hline
\end{tabular}

Notes. The "Short" column indicates configuration pairs requiring a single time interval for a changeover, whereas the pairs in the "Long" column require three time intervals. 
often referred to as a metroplex of airports. In this case it is not sufficient, in general, to optimize each airport separately, because one might end up with an infeasible solution for the system as a whole due to the interactions between arrivals and departures at the different airports. This relationship has been studied recently in Bonnefoy and Hansman (2005) and Bonnefoy (2008). In particular, the capacity of a metroplex as a system will in general be lower than the sum of the capacities of its individual airports.

Furthermore, the use of a given configuration at one airport will often impact the range of configurations that can be used simultaneously at neighboring airports within the metroplex. Consider, for example, the New York metroplex consisting of the airports of Newark (EWR), Kennedy (JFK), LaGuardia (LGA), Islip (ISP), and Teterboro (TEB). One of the many instances of the interactions mentioned above is that when JFK operates with landings on Runway 13L in IMC, LGA must also use its Runway 13 for landings and must also coordinate departures on either its Runway 4 or its Runway 13 with JFK (New York ARTCC 2008).

\subsection{Multiple Airport Mixed Integer Programming Model}

In extending the mixed integer programming model to this case, an extra index is added to each variable, corresponding to the relevant airport $p \in \mathscr{P}$, and the constraints and definitions of sets and parameters are modified accordingly. In addition, we return to the original assumption of constant changeover times across all airports. The resulting mixed integer program is presented below and can be viewed as a larger version of the single airport case, with several coupling constraints added.

$\mathbf{P 4}$

$$
\begin{array}{ll}
\min & \sum_{p \in \mathscr{P}} \sum_{t \in \mathscr{T}}\left(c_{p t} u_{p t}+q_{p t} v_{p t}\right) \\
\text { s/t. } & u_{p t}-u_{p, t-1}+\sum_{k \in \mathscr{H}_{p t}} y_{p k t}=a_{p t}, \\
& \forall p \in \mathscr{P}, \forall t \in \mathscr{T}, \\
& v_{p t}-v_{p, t-1}+\sum_{k \in \mathscr{H}_{p t}} x_{p k t}=d_{p t}, \\
\forall p \in \mathscr{P}, \forall t \in \mathscr{T}, \\
\gamma_{p j k} y_{p k t}-\beta_{p j k} x_{p k t}-\alpha_{p j k} z_{p k t} \leqslant 0, \\
\forall p \in \mathscr{P}, \forall j \in \mathscr{F}_{p k}^{t}, \forall k \in \mathscr{K}_{p t}, \forall t \in \mathscr{T}, \\
\gamma_{p j t}^{\prime} \sum_{k \in \mathscr{H}_{p t}} y_{p k t}-\beta_{p j t}^{\prime} \sum_{k^{\prime} \in \mathscr{H}_{p t}} x_{p k^{\prime} t} \leqslant \alpha_{p j t}^{\prime}, \\
\forall p \in \mathscr{P}, \forall j \in \mathscr{F}_{p t}^{\prime}, \forall t \in \mathscr{T}, \\
\sum_{k \in \mathscr{H}_{p t}} z_{p k t} \leqslant 1, \quad \forall p \in \mathscr{P}, \forall t \in \mathscr{T}, \\
z_{p k t}+\sum_{k^{\prime} \in \mathscr{H}_{p t-1} \backslash\{k\}} z_{p k^{\prime}, t-1} \leqslant 1, \\
\forall p \in \mathscr{P}, \forall k \in \mathscr{K}_{p t}, \forall t \in \mathscr{T} \backslash\{1\},
\end{array}
$$

$$
\begin{gathered}
z_{p k, t-1}+\sum_{k^{\prime} \in \mathscr{H}_{p t} \backslash\{k\}} z_{p k^{\prime} t} \leqslant 1, \\
\forall p \in \mathscr{P}, \forall k \in \mathscr{K}_{p t-1}, \forall t \in \mathscr{T} \backslash\{1\}, \\
z_{p k t}+z_{p^{\prime} k^{\prime} t} \leqslant 1, \quad \forall p \in \mathscr{P}, \forall k \in \mathscr{K}_{p t}, \\
\forall\left\{p^{\prime}, k^{\prime}\right\} \in \mathcal{N}_{p k}, \forall t \in \mathscr{T}, \\
\bar{\gamma}_{j}^{i} \sum_{p \in \mathscr{P}} \sum_{k \in \mathscr{H}_{p t}} y_{p k t}-\bar{\beta}_{j}^{i} \sum_{p^{\prime} \in \mathscr{P}} \sum_{k^{\prime} \in \mathscr{H}_{p^{\prime} t}} x_{p^{\prime} k^{\prime} t} \leqslant \bar{\alpha}_{j}^{i}, \\
\forall i \in \mathscr{F}, \forall j \in \overline{\mathscr{F}}_{i}, \forall t \in \mathscr{T}, \\
z_{p k t} \in\{0,1\}, \quad \forall p \in \mathscr{P}, \\
u_{p t}, v_{p t} \in \mathbb{Z}_{+}, \quad \forall p \in \mathscr{P}, \forall t \in \mathscr{T}, \\
y_{p k t} \geqslant 0, \quad \forall p \in \mathscr{P}, \forall k \in \mathscr{K}_{p t}, \forall t \in \mathscr{T}, \\
x_{p k t} \geqslant 0, \quad \forall p \in \mathscr{P}, \forall k \in \mathscr{K}_{p t}, \forall t \in \mathscr{T}, \\
u_{p 0}, v_{p 0}=0, \quad \forall p \in \mathscr{P} .
\end{gathered}
$$

Addressing first the similarities of $\mathrm{P} 4$ with $\mathrm{P} 1$, our objective is again to minimize the total cost of delays incurred, where $u_{p t}$ and $v_{p t}$ are the number of arrivals and departures, respectively, that go unserved at airport $p$ at time $t$. These variables are defined by Constraints (33), (34), (43), and (46). Constraints (35) force the operating point, $\left(x_{p k t}, y_{p k t}\right)$, given that we operate in the $(p, k)$ th RCCE at time $t$, to lie within this RCCE, while forcing it to zero if $z_{p k t}=0$, in which case we do not operate in RCCE $(p, k)$ at time $t$. Constraints (37) state that, at any time $t$, we may operate in at most one configuration at each airport, while Constraints (38) model the cost of changing from one configuration to another at each airport by enforcing a delay of one time interval. Finally, for a fixed airport $p$, Inequalities (36) are identical to Inequalities (12), which were added to P1; and similarly, Inequalities (39) are the same as Inequalities (11), overlooking the notational difference of an extra $p$ in the subscripts.

In addition to these constraints, which are in essence the same in $\mathrm{P} 4$ as in P1, we model the interdependence between airports in Constraints (40) and (41) as follows. Letting $\mathcal{N}_{p k}=\left\{\left\{p_{1}^{\prime}, k_{1}^{\prime}\right\},\left\{p_{2}^{\prime}, k_{2}^{\prime}\right\}, \ldots\right\}$ be the set of pairs such that we cannot operate in configuration $k$ at airport $p$ while also operating in configuration $k^{\prime}$ at airport $p^{\prime}$, for any $\left\{p^{\prime}, k^{\prime}\right\} \in \mathcal{N}_{p k}$, Constraints (40) state that we cannot operate in configurations at different airports that are incompatible. In Constraints (41), we assume that for each index $i$ in some set $\mathscr{F}$, we have a system-wide capacity envelope in effect at every time interval that models the extra dependence between airports, and let $\bar{\alpha}_{j}^{i}, \bar{\beta}_{j}^{i}$, and $\bar{\gamma}_{j}^{i}$ be the parameters of the $j$ th piece of the $i$ th capacity envelope, where $j \in \bar{y}_{i}$. Indeed, the dependence might be much deeper than this, ideally requiring capacity envelopes for every combination of airports and configurations. However, such envelopes might be difficult to obtain, requiring sophisticated computational modeling of the system, whereas the 
system-wide envelopes above might be obtained through observed system data.

\subsection{Computational Results}

We show here a large example with 5 airports, which have, respectively, 10, 8, 4, 4, and 4 configurations and 26, 20, 11,10 , and 12 RCCE, giving a total of 30 configurations and 79 RCCE. Problem characteristics are varied, and computational results are presented in Tables 9 and 10 .

In Table 9, when system-wide capacity envelopes, Constraints (41), are omitted, one can observe that the solution times are highly variable, depending on the characteristics of the problem. In varying the characteristics, we start by solving the full problem (i.e., with all configurations and RCCE available and all combinations allowed) and then modify the problem so that the optimal solution becomes infeasible. Proceeding in this manner, one can see that disallowing certain combinations of configurations being used between airports reduces computation time, while removing the availability of certain RCCE and configurations at certain times can greatly increase computation time.

These trends make sense because disallowing combinations of configurations between airports reduces problem size, while progressively removing optimal solutions by changing the problem data is likely to result in an optimal solution that is more difficult to obtain by branch and bound. Note as well that each of the first three rows of Table 9 closely corresponds to solving five single airport problems, because there is no coupling between the five airports in these instances. This gives further evidence that the models we have presented to solve the single airport problem are tractable.

Table 10 shows computational results once a systemwide RCCE, Constraint (41), has been added. First, one should note that no optimal solutions were obtained within five minutes, and hence we present the bounds obtained. This is clearly a significantly worsened performance, compared to the single-airport case. However, it is encouraging that good feasible solutions were always obtained

Table 9. Effect of problem characteristics on computation time for the metroplex case with no system-wide RCCE.

\begin{tabular}{|c|c|c|c|c|}
\hline $\begin{array}{l}\text { Configurations } \\
\text { unavailable }{ }^{\dagger}\end{array}$ & $\begin{array}{c}\text { RCCE } \\
\text { unavailable }\end{array}$ & $\begin{array}{c}\text { Some } \\
\left\{\{p, k\},\left\{p^{\prime}, k^{\prime}\right\}\right\} \\
\text { disallowed }^{\ddagger}\end{array}$ & $\begin{array}{c}\text { Optimal } \\
\text { objective } \\
\text { value }\end{array}$ & Time (s) \\
\hline No & No & No & 28,422 & 2.6 \\
\hline Yes & No & No & 35,120 & 23.5 \\
\hline Yes & Yes & No & 36,424 & 79.6 \\
\hline No & No & Yes & 28,422 & 1.3 \\
\hline Yes & No & Yes & 35,120 & 27.3 \\
\hline Yes & Yes & Yes & 36,862 & 30.3 \\
\hline
\end{tabular}

Table 10. Effect of problem characteristics on the bounds on the optimal objective value obtained within five minutes for the metroplex case with a system-wide RCCE.

\begin{tabular}{lcccc}
\hline $\begin{array}{l}\text { Configurations } \\
\text { unavailable }^{\dagger}\end{array}$ & $\begin{array}{c}\text { RCCE } \\
\text { unavailable }\end{array}$ & $\begin{array}{c}\text { Some }\{\{p, k\}, \\
\left.\left\{p^{\prime}, k^{\prime}\right\}\right\} \\
\text { disallowed }^{\ddagger}\end{array}$ & $\begin{array}{c}\text { Bounds on } \\
\text { optimal } \\
\text { objective value }\end{array}$ & $\begin{array}{c}\text { Optimality } \\
\text { gap (\%) }\end{array}$ \\
\hline No & No & No & $(83,397,83,904)$ & 0.6 \\
Yes & No & No & $(83,397,85,744)$ & 2.7 \\
Yes & Yes & No & $(83,397,84,342)$ & 1.1 \\
No & No & Yes & $(83,397,83,896)$ & 0.6 \\
Yes & No & Yes & $(83,397,85,206)$ & 2.1 \\
Yes & Yes & Yes & $(83,397,84,220)$ & 1.0 \\
\hline
\end{tabular}

$\dagger(1,4,13),(1,9,20),(2,2,13),(2,4,20),(3,3,13),(4,2,5),(4,2,6)$

$\ddagger\{\{1,9\},\{2,3\}\},\{\{2,3\},\{4,4\}\},\{\{3,2\},\{5,4\}\}$.

within one minute and that the best solutions obtained after five minutes typically were within about $2 \%$ of optimality. In addition, observe that in this example the system-wide RCCE is quite restrictive given the increase in the optimal objective values that can be inferred from comparing Tables 9 and 10.

It is evident that the addition of a system-wide RCCE has a significant effect on computation time, and that as a result the metroplex formulation is less effective than the single airport formulation. Nevertheless, good solutions are obtained within a practical amount of time.

\section{Further Extensions}

We consider next extensions to the basic model that address issues occasionally arising in practice. Suppose that there are certain environmental constraints on certain configurations, such as federal or local regulations (or, in many cases, local "letters of understanding") governing airport operations. For example, the use of a configuration that leads to departures taking off over a residential area might be prohibited or discouraged during certain times of the day. We show here that the model can be easily extended to accommodate such restrictions.

Consider, for example, the following cases for the single airport problem:

1. The maximum total operating time in configuration $k$ is $S_{1}$ time intervals in any continuous period of length $S_{2}>S_{1}$. We add the following $T+1-S_{2}$ constraints:

$$
\begin{aligned}
\sum_{t \in \mathscr{Q}} z_{k t} \leqslant S_{1}, \quad \forall t^{\prime} & \in\left\{1,2, \ldots, T+1-S_{2}\right\}, \\
\text { where } \mathscr{Q} & =\left\{t^{\prime}, t^{\prime}+1, \ldots, t^{\prime}+S_{2}-1\right\} \subset \mathcal{T} .
\end{aligned}
$$

2. Once we operate in configuration $k$, and then stop, we may not resume operation in this configuration until it has been inoperative for $S$ time intervals. Assuming that there are at least $S$ time intervals remaining after some time $t$, we want $z_{k t}=1, z_{k, t+1}=0 \Rightarrow z_{k, t+2}, z_{k, t+3}, \ldots, z_{k, t+S}=0$. 
We add $2 T-4$ variables, $w_{t}$ and $\psi_{t}$, and the following $3 T-6+(T-S)(S-1)+(S-2)(S-1) / 2$ constraints to our model:

$w_{t} \geqslant 0, \quad \forall t \in\{1,2, \ldots, T-2\}$,

$w_{t} \geqslant z_{k t}-z_{k, t+1}, \quad \forall t \in\{1,2, \ldots, T-2\}$,

$\psi_{t} \geqslant z_{k, t+j+1}, \quad \forall t \in\{1,2, \ldots, T-2\}, \forall j \in\left\{1,2, \ldots, s^{\prime}\right\}$,

where $s^{\prime}=\min \{S-1, T-t-1\}$,

$\psi_{t}+w_{t} \leqslant 1, \quad \forall t \in\{1,2, \ldots, T-2\}$.

Note from Constraints (49) that $w_{t} \geqslant 1$ if we operate in the $k$ th configuration at time $t$ but not at time $t+1$, and from Constraints (50) that $\psi_{t} \geqslant 1$ if we operate in configuration $k$ in any of the $s^{\prime}$ time intervals after interval $t+1$. Constraints (51) then state that at most one of these events can occur.

Given that such extensions would normally complicate the decision-making process significantly, the ease with which the MIP models can incorporate them is an important benefit.

\section{Conclusions}

We have presented a strong mixed integer programming formulation to solve the single airport RCM and ADRB problems. Evidence provided in the form of computational results on realistic problem data indicates that our model is capable of solving real-world instances to optimality quickly enough to be implemented. To obtain a lower bound on the expected benefits from our optimization approach, a sophisticated optimization-based heuristic was developed that reveals the potential cost savings to be indeed significant. It was also shown that a number of additional potential constraints and local considerations can be incorporated into the models with little difficulty. Finally, we have also proposed an extension of the model to optimize over a metroplex of airports. Although the results reported herein are encouraging, this is a topic that lends itself to much future work.

We have now started, in association with the MIT Lincoln Laboratory, a process that might eventually lead to field implementation of our approach. As a first step, the optimization models would be operated "offline" for one or more selected airports and their recommendations, and attendant delays would be compared with the actual sequences selected by the FAA and the observed delays to arrivals and departures. If successful, we envisage that in the long run the approach will be implemented using a rolling horizon, with the solution being updated at relatively short intervals (e.g., every 30 minutes) to compensate for the uncertainty in some of the data.

\section{Acknowledgments}

The authors thank Bill Moser of MIT Lincoln Laboratory and Tom Reynolds of MIT Partnership for Air Transportation Noise and Emissions Reduction and of MIT Lincoln Laboratory for helpful discussions. This work was partially supported by NSF grant EFRI-0735905 and the MIT Lincoln Laboratory.

\section{References}

Bonnefoy, P. 2008. Scalability of the air transportation system and development of multi-airport systems: A worldwide perspective. Ph.D. thesis, Engineering Systems Division, Massachusetts Institute of Technology, Cambridge, MA

Bonnefoy, P., R. J. Hansman. 2005. Emergence and impact of secondary airports in the United States. FAA/Eurocontrol Air Traffic Management Conf. (ATM), Baltimore.

de Neufville, R., A. Odoni. 2003. Airport Systems: Planning, Design, and Management. McGraw-Hill, New York.

Gilbo, E. P. 1993. Airport capacity: Representation, estimation, optimization. IEEE Trans. Control Systems Tech. 1(3) 144-154.

Gilbo, E. P. 1997. Optimizing airport capacity utilization in air traffic flow management subject to constraints at arrival and departure fixes. IEEE Trans. Control Systems Tech. 5(5) 490-503.

Gilbo, E. P., K. W. Howard. 2000. Collaborative optimization of airport arrival and departure traffic flow management strategies for CDM. 3rd USA/Europe Air Traffic Management R\&D Seminar, Naples, Italy.

Hall, W. D. 1999. Efficient capacity allocation in a collaborative air transportation system. Ph.D. thesis, Operations Research Center, Massachusetts Institute of Technology, Cambridge, MA.

Kellner, S., D. Kösters. 2008. Contribution to the ATMAP project. Working paper, Institute of Transport Science, RWTH Aachen University, Aachen, Germany.

New York ARTCC. 2008. N90 TRACON_LaGuardia standard operating procedures. New York ARTCC, New York.

Stamatopoulos, M. A., K. G. Zografos, A. R. Odoni. 2004. A decision support system for airport strategic planning. Transportation Res. Part C 12 91-117.

Swedish, W. J. 1981. Upgraded FAA airfield capacity model. Report FAAEM-81-1, Vols. 1 and 2, The MITRE Corporation, McLean, VA. 